\title{
Detecting and refactoring code smells in spreadsheet formulas
}

\author{
Felienne Hermans • Martin Pinzger • Arie van Deursen
}

(C) Springer Science+Business Media New York 2014

\begin{abstract}
Spreadsheets are used extensively in business processes around the world and just like software, spreadsheets are changed throughout their lifetime causing understandability and maintainability issues. This paper adapts known code smells to spreadsheet formulas. To that end we present a list of metrics by which we can detect smelly formulas; a visualization technique to highlight these formulas in spreadsheets and a method to automatically suggest refactorings to resolve smells. We implemented the metrics, visualization and refactoring suggestions techniques in a prototype tool and evaluated our approach in three studies. Firstly, we analyze the EUSES spreadsheet corpus, to study the occurrence of the formula smells. Secondly, we analyze ten real life spreadsheets, and interview the spreadsheet owners about the identified smells. Finally, we generate refactoring suggestions for those ten spreadsheets and study the implications. The results of these evaluations indicate that formula smells are common, that they can reveal real errors and weaknesses in spreadsheet formulas and that in simple cases they can be refactored.
\end{abstract}

Keywords Spreadsheets $\cdot$ Code smells $\cdot$ Refactoring

\section{Introduction}

The use of spreadsheets is very common in industry, Winston (2001) estimates that $90 \%$ of all analysts in industry perform calculations in spreadsheets. Spreadsheet developers are in fact end-user programmers that are usually not formally trained software engineers. There

Communicated by: Massimiliano Di Penta and Jonathan Maletic

F. Hermans $(\bowtie) \cdot$ M. Pinzger · A. van Deursen

Delft University of Technology, Mekelweg 4, 2628 CD Delft, The Netherlands

e-mail: F.F.J.Hermans@tudelft.nl

M. Pinzger

e-mail: m.pinzger@tudelft.nl

A. van Deursen

e-mail: arie.vandeursen@tudelft.nl 
are many of those end-user programmers, more than there are traditional programmers, and the artifacts they create can be just as important to an organization as regular software. Technically, spreadsheets also have similarities to software. One could view spreadsheet formulas as little pieces of source code, since both consist of constants, variables, conditional statements and references to other parts of the software. It therefore seems viable to investigate what principles from software engineering are also applicable to spreadsheets.

In previous work (Hermans et al. 2012a) we have defined code smells between worksheets, such as high coupling between worksheets and middle men worksheets. The evaluation of those smells showed that they can indeed reveal weak spots in a spreadsheet's design. In this paper we follow that line of thought, but focus our attention on smells within spreadsheet formulas. To that end we present a set of formula smells, based on Fowler's code smells. We subsequently define metrics for each of the formula smells, to enable the automatic detection of the smells. Our detection approach uses thresholds to divide the severity of each formula smell into low, moderate, and high. The thresholds are based on the analysis of 4,233 spreadsheets from the EUSES corpus (Fisher and Rothermel 2005). Thereon we address the issue of communicating the detected smells to spreadsheet users. We choose to do this within the spreadsheet itself, with a spreadsheet risk map, a colored overlay on the spreadsheet, indicating risk in the spreadsheet formulas. Finally we evaluate the catalog of smells in two ways, with a quantitative and qualitative evaluation. First, we perform a quantitative evaluation on the EUSES spreadsheet corpus. Then, a qualitative analysis was performed with ten real life spreadsheets and their developers from industry. With these two studies we aim to answer the three research questions

$R_{1} \quad$ What formula smells are most common, and why?

$R_{2} \quad$ To what extent do formula smells expose threats to spreadsheet quality?

$R_{3}$ To what extent are risk maps an appropriate way to visualize formula smells?

This paper is an extension of our previous paper published in the proceedings of ICSM 2012 (Hermans et al. 2012b). In comparison to the ICSM paper, this paper adds: an extended version of the discussion section (Section 13); a broader overview of related work (Section 14); a catalog of refactorings to resolve the formula smells (Section 4); a description of an implementation that generates refactorings (Section 6.2) and a new exploratory study of the impact of refactoring smells. This additional study, found in Section 11, answers the additional research question

$R_{4} \quad$ To what extent can formula smells be refactored by generated refactoring suggestions?

The aim of this extended version is to gain a more deeper understanding on how detected smells in spreadsheets can be refactored. The evaluations in the original ICSM paper showed that formula smells can indeed reveal weaknesses and even find real mistakes in a spreadsheet. It furthermore showed that risk maps, although not yet perfect, are a good aid in locating and understanding formula smells. Based on the additional evidence we present in this paper, we conclude that many spreadsheet smells can be refactored; however, some smells are in balance with each other and additional support is needed to help users find the sweet spot in that balance.

\section{Formula Smells}

In this section, we present our formula smells, which are based on the existing work in the field of source code smells. Smells in source code indicate suspicious pieces of 
code, that the developer might want to refactor to improve readability and minimize the chance of future errors. Formula smells are inspired by source code smells: they indicate formulas that are suspicious; not easy to read or error-prone. To create our catalog of formula smells, we looked at all inter-class code smells defined by Fowler (1999) and removed those code smells that are specific to source code, like the Temporary Field smell. This left us with the following list of formula smells. Note that in other work (Hermans et al. 2012a), we studied code smells between worksheets, based on code smells between classes.

\subsection{Multiple Operations}

One of the most well-known code smells is the Long Method. Inspired by this code smell, we define the formula smell Multiple Operations. Analogous to a long method, a formula with many operations will likely be harder to understand than a shorter one. Especially since in most spreadsheet programs, there is limited space to view a formula, causing long formulas to be cut off.

\subsection{Multiple References}

Another code smell that we use as a basis is the Many Parameters code smell. A method that uses many input values might be split into multiple methods to improve readability. The formula equivalent of this smell occurs when a formula references many different other cells, like $S U M(A 1: A 5 ; B 7 ; C 18 ; C 19 ; F 19)$. In this case the essence of the formula is clear; some cells are summed. However locating the different cells that are contained in the sum can be challenging.

\subsection{Conditional Complexity}

Fowler states that the nesting of many conditional operations should be considered a threat to code readability, since conditionals are hard to read. Since spreadsheet systems also allow for the use of conditionals, spreadsheet formulas are at risk of this treat too. We hence consider a formula with many conditional operations as smelly, like the formula $\operatorname{IF}(A 3=1, \operatorname{IF}(A 4=1, \operatorname{IF}(A 5<34700,50)), 0)$. Because this formula contains many conditional branches, it is quite hard to read.

\subsection{Long Calculation Chain}

Spreadsheet formulas can refer to each other, creating a chain of calculations. In Fig. 1 a spreadsheet is shown in which we find a calculation chain of two steps: C15 refers to F3, G3 and B15. G3 and B15 are constants, F3 however refers to B3 to E3. To fully understand what a chain of formulas calculates, a spreadsheet user has to trace along all steps to find the origin. Nardi and Miller (1990) described that spreadsheet users find tracing a long calculation chain a tedious task. Note that there is a trade off between this metric on the one hand and Multiple Operations and Multiple References on the other. When they are lowered, this metric will be increased, and vice versa. Such trade offs occur in source code smells too. 


\begin{tabular}{|c|c|c|c|c|c|c|c|c|}
\hline C15 & \multirow{2}{*}{$\frac{T}{\mathrm{~A}}=$} & \multirow[t]{2}{*}{$f_{x}$} & \multicolumn{6}{|c|}{$=(F 3+G 3)^{*} B 15$} \\
\hline 4 & & & $\mathrm{C}$ & D & $E$ & $\mathrm{~F}$ & G & \\
\hline 1 & \multicolumn{5}{|c|}{ Hours Worked in December } & & \multirow[b]{2}{*}{ Overtime } & \\
\hline 2 & & week 1 & week 2 & week 3 & week 4 & \multirow{2}{*}{ Total Hours } & & \\
\hline 3 & Green & 20 & 34 & 25 & 20.3 & & 0 & \\
\hline 4 & Jones & 35 & 34 & 33 & 34 & 136 & 0 & \\
\hline 5 & Smith & 25 & 26 & 27 & 26 & 104 & 0 & \\
\hline 6 & Johnson & 28 & 30 & 21 & 6.3 & 105.3 & 0 & \\
\hline 7 & White & 45 & 10 & 14 & 23 & 92 & 5 & \\
\hline 8 & Edwards & 37 & 38 & & 38.3 & 153.3 & 0 & \\
\hline \multicolumn{9}{|l|}{9} \\
\hline \multicolumn{8}{|l|}{10} & \\
\hline 11 & Weekly Total & 193 & & 70 & 173.9 & 695.9 & 5 & \\
\hline 12 & Max Hours & 45 & & 40 & 38.3 & 153.3 & 5 & \\
\hline \multicolumn{8}{|l|}{13} & \\
\hline 14 & & Hourly & Gross Pay & & & & & \\
\hline 15 & Green & $\bullet \quad 15$ & $58,579.50$ & & & & & \\
\hline 16 & Jones & 17 & $\$ 2,312.00$ & & & & & \\
\hline 17 & Smith & 18 & $\$ 1,872.00$ & & & & & \\
\hline 18 & Johnson & 14 & $\$ 1,474.20$ & & & & & \\
\hline 19 & White & 14 & $\$ 1,358.00$ & & & & & \\
\hline 20 & \multirow[t]{2}{*}{ Edwards } & 20 & $\$ 3,066.00$ & & & & & \\
\hline 21 & & & & & & & & \\
\hline
\end{tabular}

Fig. 1 A spreadsheet containing a calculation chain of two steps

\subsection{Duplicated Formulas}

Finally there is the duplication code smell, that indicates that similar snippets of code are used throughout a class. This is a concept common in spreadsheets too, where some formulas are partly the same as others. Consider, for example, a worksheet that contains a cell with formula $\operatorname{MIN}(A 1: A 6)+10 \%$ and a second formula $M I N(A 1: A 6)+20 \%$. This formula exhibits duplication; the part $M I N(A 1: A 6)$ is contained in more than one formula. A group of formulas that exhibit duplication can be found in Fig. 2. Duplication is suspicious for two reasons. Firstly it poses a threat to maintainability, since when the duplicated part of the formula is adapted, this adaptation has to be performed at multiple places. This could be forgotten by the spreadsheet user, or a mistake could be made in some of the cases. Secondly, there is an impact on readability, when long parts of the formula are duplicated, it is hard to see how they differ from each other.

\section{Formula Smell Metrics}

To identify smell occurrences automatically, we make use of metrics. This is a common approach in the automatic detection of code smells (Moha et al. 2010). We follow our approach outlined in Hermans et al. (2012a) defining a metric to detect each of the formula

Fig. 2 Formulas containing similar subtrees

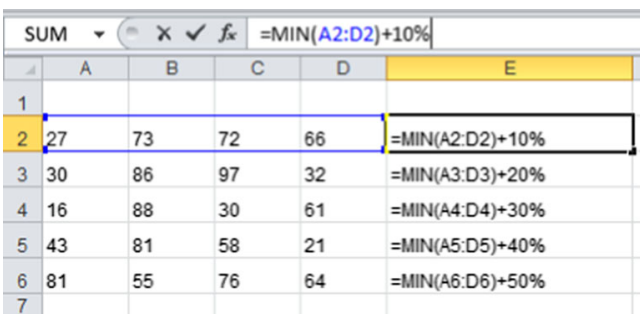


smells in spreadsheets. This method entails the definition of a metric for each of the smells to indicate the presence of that particular smell.

Multiple Operations We measure the length of a formula in terms of the total number of functions that the formula contains.

Multiple References This metric is counted in terms of the number of ranges a formula is referring to. We use the number of ranges rather than the number of individual references, as in previous work we have found that formulas referring to multiple ranges are more difficult for spreadsheet users to read (Hermans et al. 2012c).

Conditional Complexity is measured in terms of the number of conditionals contained by a formula.

Long Calculation Chain This metric is defined as the length of the longest path of cells that need to be dereferenced when computing the value of the formula.

Duplicated Formula For the localization of this smell more information about all formulas in the spreadsheet is needed. Consider the spreadsheet in Fig. 2. All the formulas in column E calculate the minimum of the four cells left to it, followed by the addition of a certain percentage. We argue that in this case, duplication should be detected. However, looking at the formulas, they do not look similar. We therefore will use the relative RIC1 notation when detecting duplication.

In the relative $\mathrm{R} 1 \mathrm{C} 1$ notation, references to other cells are expressed relative to the cell containing the formula. $M I N(A 2: D 2)$ in cell E2 is written as $M I N(R C[-4]: R C[-1])$ in relative R1C1 notation. With this notation, all formulas in Fig. 2 contain the subformula $\operatorname{MIN}(R C[-4]: R C[-1])$, with different percentages. With this, we will measure the duplicate code smell as the number of formulas, located in the same worksheet and expressed in relative $\mathrm{R} 1 \mathrm{C} 1$ notation, with which a formula shares at least one proper subtree. We exclude the entire tree as subtree, since having the same $\mathrm{R} 1 \mathrm{C} 1$ formula in an entire row or column is the usual way of defining a formula in spreadsheets.

With our definition of smells, we focus at smells in the calculation design of the formulas. Therefore, other plausible smells, like the occurrence of magic numbers in formulas, are left out of the scope of this paper.

\section{Refactorings Corresponding to Smells}

In this section, we will describe how the five formula smells can be refactored.

\subsection{Multiple Operations}

A refactoring corresponding to the Multiple Operations smell is the division of the multiple operations over different cells in the spreadsheet. This refactoring looks at arguments of the root of the formula. Each argument of the root will be a new formula and the current formula will reference those new formulas. We call this refactoring the Extract Subformula refactoring. An additional, supporting refactoring that can be performed is the Extract Common Subformula refactoring. This refactoring can be applied if a formula contains the same subformula multiple times. 


\subsection{Multiple References}

When a formula suffers from the Multiple References smell, there are several options to refactor. The simplest refactoring we can apply, is to restructure the cells a formula references into a lower number of ranges. This is possible when adjacent cells are referenced individually, like in formula $S U M(A 1 ; A 2 ; A 3 ; A 4 ; A 5)$, this can be rewritten to $S U M(A 1: A 5)$. We call this refactoring Group References.

Secondly, there is the option to relocate the cells in the spreadsheet. If a formula is referring to multiple cells, like $S U M(A 1: A 5 ; B 7 ; C 18 ; C 19 ; F 19)$, we could move the values in B7, $\mathrm{C} 18, \mathrm{C} 19$ and F19 to A6 until A10, and subsequently rewrite the formula as $S U M(A 1: A 10)$. We call this refactoring the Move References refactoring. However, this is not always a desired option, as there might be other formulas referring to a subset of these cells. In that case, this refactoring will cause those formulas to start suffering from the Multiple References smell. Finally, we could apply the Extract Subformula refactoring. This refactoring is always applicable.

\subsection{Conditional Complexity}

To reduce the Conditional Complexity smell, in some cases we could combine multiple branches of an IFformula into one. This is possible if multiple branches result in the same value. For instance, in the formula $\operatorname{IF}(\mathrm{A} 1=5$, “OK", $\operatorname{IF}(\mathrm{B} 1=7$, "OK", "NOT OK")), two different branches result in "OK". These can be combined into one: $\operatorname{IF}(\mathrm{OR}(\mathrm{A} 1=5, \mathrm{~B} 1=7)$, "OK", "NOT OK"). This formula might be easier to understand, as it only contains one IF. If this refactoring results in a too complex condition, this can be again refactored with the Extract Subformula refactoring. We call this refactoring the Merge Branches refactoring.

If the conditional formula contains similar subformulas, the Extract Common Subformula is suggested. If that is not possible, the Extract Subformula can be applied, both on the condition and on the branches.

\subsection{Long Calculation Chain}

This smell is most difficult to refactor, as there is a trade off between this metric and Multiple Operations and Multiple References. For this smell, we look whether there are calculation steps in the chain that do not occur in other chains. If this is the case, these two calculation steps can be put into one formula. We call this refactoring the Merge Formulas refactoring. This is the only refactoring in our catalog applicable to cells with the Long Calculation Chain smell.

\subsection{Duplicated Formulas}

The Duplicated Formula smell can be refactored by putting the shared subtrees in a separate formula and replacing the subtree with a reference to that formula. This is just a special application of the Extract Subformula refactoring, in this case applied to a group of cells. In the example of Fig. 2 this would mean that column E becomes MIN(A2:D2). Then, in column E, all cells are R1C1 equal, whereas in column F we would get $\mathrm{A} 2+10 \%$. This way the common functionality is in one place $(\mathrm{E})$ and the variable part has its own place (F). If something were to change in the refactored version of the spreadsheet (for instance, MIN becomes MAX), we can adapt cell E2 and let Excel change the entire column by double 
clicking the bottom right corner of the cell. In the original situation, that change would involve updating all cells separately.

\section{Determining Smell Thresholds}

In order to use metrics as smell indicators, we determine thresholds for each of the metrics. We do this by analyzing a large body of spreadsheets. Then, based on the values for the metrics we find in that body of spreadsheets, setting the thresholds for the metrics that indicate the smell.

The body of spreadsheets we use is the EUSES Spreadsheet Corpus (Fisher and Rothermel 2005). This corpus consists of 4,223 real life spreadsheets, from all sorts of domains, ranging from educational to financial, and from inventory to biology. It was created in 2005 and has since then been used by several researchers to evaluate spreadsheet algorithms, for instance (Abraham and Erwig 2006; Cunha et al. 2009).

The total of 4,223 spreadsheets together contain 15,015 worksheets and 426,986 formulas. A spreadsheet however often contains many rows with formulas that are equal in the relative R1C1 notation, which we call unique formulas. We collect the metrics for all unique formulas, of which there are 55,736 in the EUSES corpus. Figure 3 shows the cumulative percentage of formulas (x-axis) above a given metric value (y-axis) for all unique formulas in the EUSES corpus. As can been seen in this figure, the metrics all follow a power law like distribution, having most of their variability on the tail.

We therefore choose to select the thresholds in this tail. In the analysis of source code smells (Alves et al. 2010), the percentages 70, 80 and $90 \%$ are common thresholds and looking at the distribution, we propose to use these thresholds for formula smells too. Hence, the values at 70,80 and $90 \%$ of the metric values will correspond to the risk levels low, moderate and high. In the subsequent two evaluations, we will investigate whether these thresholds are well chosen.

Table 1 shows the thresholds that follow from the given selection for the five formula smells.

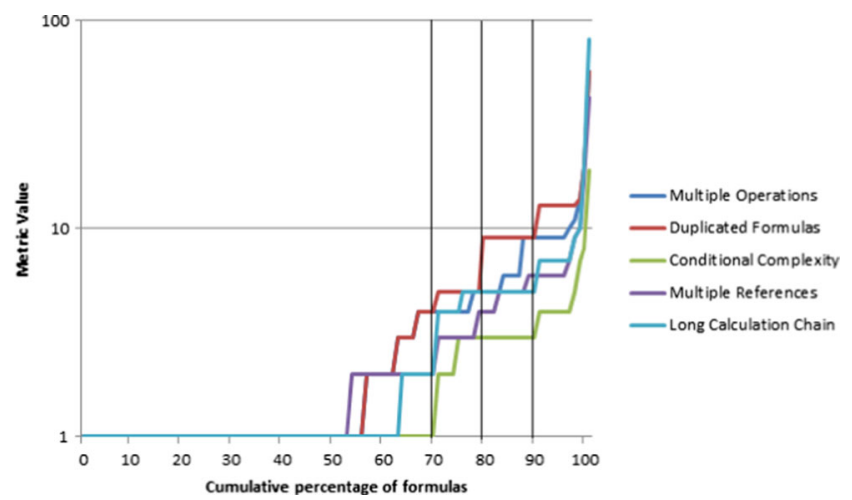

Fig. 3 A quantile plot (\% of formulas) for the five metrics for formulas in the EUSES corpus, with a logarithmic scale for the $\mathrm{y}$ axis 
Table 1 Thresholds for the metrics that indicate the formula smells

\begin{tabular}{llll}
\hline Smell & $70 \%$ & $80 \%$ & $90 \%$ \\
\hline Multiple operations & 4 & 5 & 9 \\
Multiple references & 3 & 4 & 6 \\
Conditional complexity & 2 & 3 & 4 \\
Long calculation chain & 4 & 5 & 7 \\
Duplicated formulas & 6 & 9 & 13 \\
\hline
\end{tabular}

\section{Risk Maps}

Now we have established a method to detect the formula smells, this section will describe a way to communicate the located smells and their corresponding refactorings to spreadsheet users.

\subsection{Smells}

We have chosen to show the formula smells inside the spreadsheet itself. We have seen in previous work that, when reasoning about formulas, spreadsheet users like to see the context of the formula (Hermans et al. 2012a). We therefore use a colored overlay over the spreadsheet that indicates the formula smells, inspired by other spreadsheet tools like UCheck (Abraham and Erwig 2007; Chambers and Erwig 2009; Erwig 2009), combined with pop-ups showing what smell is detected, similar to those in UFix (Abraham and Erwig 2005). We call this the spreadsheet risk map. Before the risk map is generated, existing colors are removed from the spreadsheet.

We attach a comment to each colored cell, so when a spreadsheet user clicks a cell, an explanation about the located smell will pop up. The three different risk levels are marked with different colors; yellow for low; orange for moderate and red for high. If multiple smells are detected in one cell, they are both shown and ordered by severity. The color of the cell represents the severity of the worst smell. For instance, the pop-up in Fig. 4 shows that cell AQ3 suffers from both the Multiple Operations and Multiple References smells, the red color of the cell indicates the worst smell is detected at the $90 \%$ level.

\subsection{Refactoring}

At this point, we do not yet offer support for automated refactoring. We generate suggestions for refactoring and let the spreadsheet users execute them themselves. Generation of the suggestions is done by trying to apply the refactorings on smelly formulas in the order as

\begin{tabular}{|c|c|c|c|c|c|c|c|c|c|c|c|c|c|}
\hline \multicolumn{2}{|r|}{$\mathrm{AQ} 3$} & \multicolumn{2}{|l|}{-} & \multicolumn{10}{|c|}{ =AP3-MIN(R3:AO3)-SMALL(R3:A03;2)-SMALL(R3:AO3;3)-SMAL(R3:A03;4)-SMALL(R3:AO3;5) } \\
\hline 4 & A & AN & AO & AP & $A Q$ & AR & AS & AT & AU & AY & BB & BC & BD \\
\hline 1 & Code & Quiz & Quiz & Quiz & Drop & Quiz & Quiz & Exam & Exam & Final & Term & Total & \\
\hline 2 & & 23 & 24 & Total & 5 & Tinis & 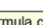 & thins m & ny & & Paper & Grade & \\
\hline 3 & & 10 & 10 & 240 & 190 & $10 \mathrm{op}$ & 25 ( $\mathrm{Hi}$ & & $\%$ & $100 \%$ & $100,0 \%$ & $100,0 \%$ & Grade \\
\hline 4 & 030767 & 10 & 5 & 166 & 163 & 8 This $\mathrm{f}$ & rmula r & ers to man & ny 6 & $87 \%$ & $96,5 \%$ & $87,7 \%$ & $B+$ \\
\hline 5 & 11291129 & 9 & 10 & 155 & 152 & 8 group & of cells & (High) & 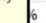 & $71 \%$ & $95,0 \%$ & $82.4 \%$ & B \\
\hline 6 & 12345 & 9 & 10 & 211 & 187 & 96,470 & 50,4 & $94 \pi$ & \% & $89 \%$ & $100,0 \%$ & $96,0 \%$ & A \\
\hline 7 & 2182 & 10 & 10 & 164 & 158 & $83,2 \%$ & 83,2 & $88 \%$ & $94 \%$ & $99 \%$ & $98,0 \%$ & $93,4 \%$ & A \\
\hline 8 & 2663 & 10 & 10 & 200 & 185 & $97,4 \%$ & 97,4 & $97 \%$ & $71 \%$ & $86 \%$ & $98,0 \%$ & $91,2 \%$ & A- \\
\hline 9 & 3196 & 10 & 10 & 203 & 181 & $95,3 \%$ & 95,3 & $88 \%$ & $90 \%$ & $93 \%$ & $100,0 \%$ & $92,5 \%$ & A \\
\hline
\end{tabular}

Fig. 4 A spreadsheet with its risk map 


\begin{tabular}{|c|c|c|c|c|}
\hline \multicolumn{2}{|c|}{ B13 } & \multicolumn{3}{|l|}{$=$ SUM(B2:B4)-SUM(B7:B11) } \\
\hline 4 & A & B & C & D \\
\hline 1 & Profit and Loss & 2011 & & \\
\hline 2 & Total Europe & $=$ Turnover!C7 & & \\
\hline 3 & Total Asia & =Turnover!C15 & & \\
\hline 4 & Total USA & =Turnover!C16 & & \\
\hline 5 & Total Turnover & $=\operatorname{SUM}(B 2: B 4)$ & & \\
\hline 6 & Costs & 2011 & & \\
\hline 7 & Housing & $=$ Costs $! C 7$ & & \\
\hline 8 & Equipment & $=$ Costs $! \mathrm{C} 12$ & & \\
\hline 9 & Salary director & $=$ Costs $! \mathrm{C} 17$ & & \\
\hline 10 & Salary employees & $=$ Costs $! C 18$ & \multirow{3}{*}{$\begin{array}{l}\text { Refactoring Possible: } \\
\text { Common Subformula } \\
\text { SUM(B2:B4) can be } \\
\text { extracted. }\end{array}$} & \\
\hline 11 & Other & $=$ Costs $! \mathrm{C} 20$ & & \\
\hline 12 & Total Costs & $=\operatorname{SUM}(B 7: B 11)$ & & \\
\hline 13 & EBITA & ESUM(B2:B4)-SUM(B7:B11) & & \\
\hline 14 & & & & \\
\hline
\end{tabular}

Fig. 5 A spreadsheet with a refactoring suggestion. The subformula SUM(B2:B4) also occurs in B5 and could be extracted from the highlighted formula in B13

described in Section 4. In the suggestion that is generated, we place the suggestions for the refactorings in the pop-ups that also contain the smells, as illustrated by Fig. 5.

Furthermore, a list is generated containing all refactorings that can be applied, to make it easier for users to check them all.

\section{Implementation}

The generation of the risk maps is incorporated into the existing spreadsheet analysis system Breviz (Hermans et al. 2011). Breviz is implemented in C\# 4.0. It utilizes the Gembox component to read Excel files. ${ }^{1}$ Breviz reads an Excel file and calculates the metrics described above and subsequently generates the spreadsheet risk map. Furthermore the metric values are stored in a SQL Server 2008 database to perform the threshold detection. Breviz, including refactoring suggestions, is available as a SaaS application at http://app.infotron.nl.

\section{Evaluation}

In order to evaluate the formula smells, metrics, thresholds, risk map visualization and refactoring suggestions, we perform three separate evaluations. In the first evaluation we turn our attention back to the EUSES Spreadsheet corpus. We analyze all spreadsheets in the corpus that contain formulas. On those 1711 spreadsheets, we determine the occurrence of the five spreadsheet smells. With this analysis, we seek to find a preliminary answer to research question $R_{1}$ : what formula smells are common, and why.

For the second evaluation, aimed at a deeper understanding of $R_{1}$, plus answers to $R_{2}$ and $R_{3}$, we ask ten professional spreadsheet developers for access to their real life spreadsheets. We let our tool Breviz identify possible formula smells and show the participants the generated risk map. We thereon ask the spreadsheet users to reflect on the identified spreadsheet smells, in a semi-structured interview.

Finally, to evaluate the refactoring suggestions and answer research question $R_{4}$, we generated suggestions for those ten spreadsheets, studied the results and asked the spreadsheet

\footnotetext{
${ }^{1}$ http://www.gemboxsoftware.com/spreadsheet/overview
} 
owners to comment on a number of selected refactorings. The following sections describe the three evaluations in more detail.

\section{Smell Occurrences in the EUSES Corpus}

\subsection{Goal}

The objective of the first study is to understand how common the five formula smells are, given the thresholds we have selected. While the thresholds were chosen such as percentages of formulas containing a smell, here we are interested in the distribution of smelly formulas across spreadsheets.

\subsection{Setup}

We use the Breviz tool to analyze those spreadsheets in the EUSES corpus that contain formulas and check those 1711 spreadsheets for the presence of formula smells and their severity. Per spreadsheet the tool outputs the metric values for each of the five formula smells. We use this data to analyze the distribution of the formula smells over the three metric categories; above the 70, 80 and $90 \%$ thresholds.

This evaluation tells us whether the choices we made for the thresholds are justified, as it gives us an initial idea of the distribution of the formula smells over the spreadsheets.

\subsection{Results}

Table 2 shows the results of the first evaluation. As shown in this Table, Multiple Operations and Multiple References are most common in the EUSES Corpus. This is consistent with previous spreadsheet experiments, where it has been shown that spreadsheet are often adapted by their users (Hermans et al. 2012a). In that process, spreadsheet formulas tend to get longer and more complex. As opposed to software development, where code is sometimes rewritten to improve readability or maintainability, the answers of the ten subjects of the second evaluation (see below) have taught us that this is not a common practice among spreadsheet creators. Hence, when a formula has become long and complex, it is likely to remain as is.

Third and fourth come the Duplicated Formula and Long Calculation Chain. These two smells share the property that they are not immediately visible to the spreadsheet user. In most modern spreadsheet systems, when a cell is clicked, the formula it contains is shown. However in the case of Duplicated Formula and Long Calculation Chain, the formula does

Table 2 Percentage of spreadsheets in the EUSES corpus that suffer from at least one of the five spreadsheet smells in EUSES corpus, for the three thresholds

\begin{tabular}{lccc}
\hline Smell & $>70 \%$ & $>80 \%$ & $>90 \%$ \\
\hline Multiple references & $23.8 \%$ & $18.4 \%$ & $6.3 \%$ \\
Multiple operations & $21.6 \%$ & $17.1 \%$ & $6.3 \%$ \\
Duplicated formulas & $10.8 \%$ & $7.1 \%$ & $3.7 \%$ \\
Long calculation chain & $9.0 \%$ & $7.9 \%$ & $3.3 \%$ \\
Conditional complexity & $4.4 \%$ & $3.0 \%$ & $1.1 \%$ \\
Any of the above smells & $42.7 \%$ & $31.4 \%$ & $19.7 \%$ \\
\hline
\end{tabular}


not reveal where the calculation chain of the formula ends, and with what formulas a cell shares subtrees. So it is interesting to see that around 10 percent of spreadsheets suffer from a smell that is not visible to a spreadsheet user that is not explicitly looking for it.

Conditional Complexity is the least common smell. This is surprising, since we have seen before that conditional operations are quite common in spreadsheets. We therefore dived into this phenomenon deeper. We found that of the total of 426,986 formulas in the corpus, 5,976 contain at least one conditional operation, this is $1.4 \%$ of all formulas. These formulas are divided over 380 spreadsheets, which amounts to $22.2 \%$ of the spreadsheets with formulas. We can hence state that the use of conditional operations is relatively common.

However, only 92 of the spreadsheets $(5.3 \%)$ contain formulas with more than 2 conditional operations in one formula, adding up to only 695 formulas $(0.2 \%)$. Evidently, the nesting of conditional formulas is not very common. We will hence devote attention to this fact in the second evaluation.

Regarding the thresholds, given our current choices a substantial percentage of the corpus suffers from spreadsheet smells, especially in the low category. In the second case study we will continue to investigate the thresholds, by observing how spreadsheet users from industry feel about formulas that are marked as smelly by these thresholds.

\section{Formula Smells in an Industrial Case Study}

\subsection{Goal}

The aim of our second evaluation is investigating which of the formula smells actually poses a threat to spreadsheets $\left(R_{2}\right)$, and whether risk maps help spreadsheet users find and understand formula smells $\left(R_{3}\right)$. To determine this, we have surveyed spreadsheet users and interviewed them about a spreadsheet that they often work with and that they found was large and complicated. We stated that this spreadsheet did not necessarily had to be created by them initially, but they had to have experience with changing its formulas.

\subsection{Setup}

For the second evaluation we interviewed 10 professional spreadsheet users from the financial domain. We conducted our evaluation at Robeco, a Dutch asset management company with approximately 1600 employees worldwide, and over 130 billion Euro worth of assets under management.

We invited 27 participants of a previous study performed at Robeco (Hermans et al. 2012a) to participate in this evaluation, where they were asked to provide us with a spreadsheet that they worked with regularly. We explained to participants that we were going to analyze the quality of their spreadsheet, and discuss it with them. We provided subjects with a list of the five formula smells and a short explanation of each smell, so they could study this before the experiment. We had one interview with every participant and for each of the sessions, the procedure was as follows. First we asked the subject to explain the spreadsheet to us. We then analyzed the spreadsheet and generated the spreadsheet risk map, which we showed to users within Excel. We subsequently let subjects inspect the risk map, and asked them in a semi-structured interview setup, for each of the located formula smells 1) whether they understood the smell that was identified and 2) whether they thought this smell posed a threat to spreadsheet understandability, and if yes, why, and how severe the threat was, according to them. We finally asked them how the risk map and the pop-up helped them 
in understanding the formula smells. We made notes of each of the interviews by hand and subsequently coded them.

Each of the participants was interviewed on its own, separate from other participants and studied only their own spreadsheet. The interviews were performed face to face at the Robeco headquarters, on three different days. The interviews were executed and transcribed in Dutch, so quotes in the following are translations.

\subsection{Results}

Table 3 shows an overview of the spreadsheets used in the case study in terms of the numbers of worksheets, cells, formulas, unique formulas and file size. The five final columns indicate the number of unique formulas in the spreadsheets suffering from the given smell, Multiple Operations (MO), Duplicate Formula (DF), Multiple References (MR), Conditional Complexity $(\mathrm{CoC})$ and Long Calculation Chain $(\mathrm{CaC})$. As can be seen from this table, formula smells occur frequently in the ten spreadsheets. The following describes the result of the case studies in detail.

\subsubsection{General Observations}

When confronted with the most smelly formula cells, participants often needed time to explain the formula. In all cases the participants expressed statements like "what was this formula again?" or "let me just have a quick look". A commonality among the ten cases in the study is the fact that all participants immediately recognized the impact a smelly, hence complex formula could have on spreadsheet formulas understandability. When we discussed the most complex formulas in the spreadsheet with the participants, they indicated that it was going to be very hard to understand or adapt this formula for someone else than the spreadsheet's creator.

Most participants (8) had never considered the situation where another spreadsheet user had to understand their formulas that much. A previous study by Hermans et al. confirms the importance of such transfer scenarios (Hermans et al. 2012a). What we found in our present study, however, is that participants did not realize prior to this study that keeping formulas short and simple would help future users of the spreadsheet understand it better and faster. A side effect of our study was increased awareness with our participants that they should take understandability into account when building and adapting spreadsheets.

We found that the three thresholds and the corresponding coloring help subjects estimate severity of the detected smell. One of the subjects compared this to the triangle function in Excel. This function marks potential errors, like calculations over empty cells with a small green triangle at the bottom of a cell. He stated: "That function is annoying, since many cells get colored. Because you have different shades, I can start inspecting the red ones, and ignore the yellow ones for now".

Regarding the values of the thresholds, we discussed each colored cell with the spreadsheet owner, systematically going through the worksheets. In all but one case the subjects agreed with the classification of the formulas. Only spreadsheet user $S_{3}$ stated that he felt that the system was too strict. His spreadsheet contained 3 cells with five different references and four operations. These cells were hence marked as having both the Multiple Operations and the Multiple References smell, while the user still found this acceptable. In the other formulas in his spreadsheet where these smells were located, he did found they were smelly, since the metric values for those formulas where higher than respectively 5 and 4 . So from 


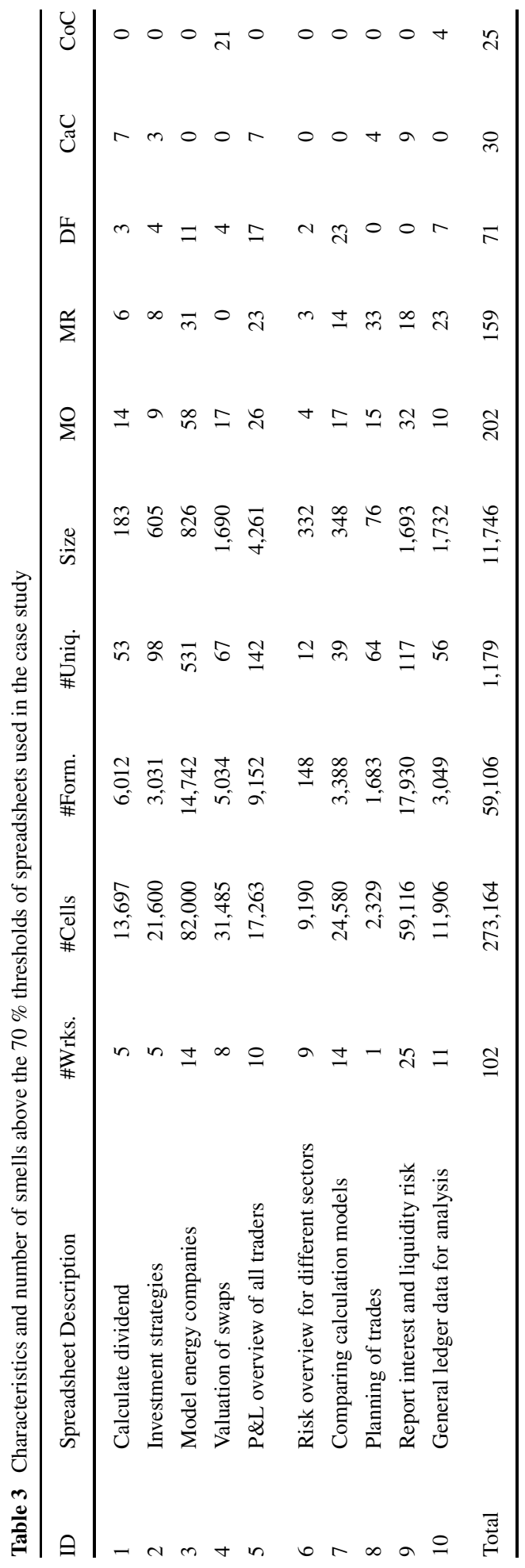


the perspective of this user the thresholds should be higher, however as stated above, he was the only one; the other nine subjects stated all marked formulas were indeed smelly.

\subsubsection{Multiple Operations}

Findings Multiple Operations were found in all ten spreadsheets, making them the number one common smell. In all cases we found that the subjects said that keeping the formulas short makes them easier to read and understand. Two of the subjects believed that formulas with many operations are often a cause of errors, saying "the chance of errors in such a long formula is so much bigger; when I find errors, it is almost always in long formulas". When asked for the reason that Multiple Operations where created, all subjects stated that this was an evolutionary process. Multiple Operations are hardly ever created at once. They are the result of the repeated adaptation of a formula, adding operations as the spreadsheet changes. As one of the subjects stated "Usually it starts with just a sum, but than you want to round it, add something and before you know it, the formula is two lines long". The two subjects above - the ones who had realized the error risk of Multiple Operations-did try to minimize formula length. However, sometimes, for instance, when a deadline was approaching, Multiple Operations were introduced anyway. There was no common practice among the spreadsheet users to restructure the spreadsheet after such a deadline. One of these two subjects mentioned "when it works, it works. No one really cares how it is done".

We found that the risk map helped in the case of Multiple Operations. In the case where this smell was located, the smelly cells were clearly marked with a color (yellow, orange or red). Hence, the reason why the smell was detected was immediately clear; many subjects stated something like "I understand why this formula is selected by your system, it is quite long."

Conclusions Spreadsheet users feel that Multiple Operations are more error prone than shorter formulas. This is especially due to the fact that formulas that suffer from the Multiple Operations smell are hard to quickly understand. Therefore, it is harder to spot an error and hence these formulas are less likely be corrected when they are wrong. Multiple Operations are often the result of changes to the spreadsheets, and the refactoring of complex formulas is not something that spreadsheet users commonly do.

\subsubsection{Multiple References}

Findings Experiences with Multiple References were similar to those with Multiple Operations; when confronted with the smelly cells, it took the nine subjects a considerable amount of time, in the longest case even ten minutes, to explain the formula. This made them realize that it would be very hard for others to understand and adapt the formula, especially since locating the references can be a challenge. Excel supports users in locating the references by coloring the referenced cells. However, if there are many references and colors users find this feature to be more annoying than helpful as confirmed by nine of our participants. One of the subjects stated, when looking at a formula that referred to no less than 17 ranges "this formula is a real puzzle".

In this case, as opposed to the Multiple Operations smell, some participants did not immediately understand how to adapt this formula to make it less smelly. When asked, one of the participants even stated "but I need all that input to make the calculation". Splitting the formula into different steps seemed more difficult than with the Multiple Operations. In that case the formulas consist of different operations, and the splitting would 
consist of separating the operations. In this case however, we encountered formulas like SUM(A1:A5;B6;D7;E12), of which it was not immediately clear to the spreadsheet users how to improve it. It can be split into multiple steps, but what steps are logical is not so easy to determine for the user. We asked the nine participants to describe how they were going to split the formula, and only one was able to formulate a strategy. The other hesitated, one of them stated "I don't know where I should start, because I don't remember how I made this formula". As an alternative, cells could be moved, such that this formula will refer to one range. This made the participants hesitate even more. They clearly felt that moving formulas around was a tricky operation, since the effect of this move on other formulas is not clear. One of the subjects that tried to lower the references said "if I move this, what will happen to the other formulas? I would like to preview that".

For this smell again, the risk maps are a good way of conveying this smell. Formulas with many references were colored red, orange or yellow; and hence attracted the attention of the spreadsheet users. Clicking the formula revealed easily that the formula had too many references.

Conclusions Subjects found that formulas with many references are not easy to understand, since finding all references can be difficult. Even though the risk was understood, subjects found it hard to come up with the right refactoring to overcome this smell. This is partly caused by the fact that a long list of references can indicate that the placement of formulas is not optimal, and hence this smell can also reveal a weakness in the organization of the spreadsheet.

Refactorings to the location of formulas were found especially hard for the subjects, and support for this, like a preview, is definitely a promising avenue for future research.

Finally we found it interesting that Excel's feature to color the cells referenced by a formula is only helpful in cases with few references (typically above 6 it got confusing for the participants). There is apparently a need for better support in locating references.

\subsubsection{Duplicated Formula}

Findings In the evaluation we found that the cases in which duplicated formulas are detected can be divided into two distinct categories.

- Common Subformula Different formulas are simply sharing a subformula, and there is an opportunity for refactoring.

- Rare Formulas: There is one formula that differs slightly from its neighbors, and therefore shares a subtree with these neighbors.

Figures 6 and 7 illustrate the two categories. In Fig. 6 the highlighted formula (in cell B13) shares the subformula $\operatorname{SUM}(B 7: B 11)$ with the formula in cell B12. The same subformula occurs twice, so it might be better to replace $\operatorname{SUM}(B 7: B 11)$ in $\mathrm{B} 13$ with a reference to B12. In Fig. 7 however something different is happening. The selected formula (E4) shares a subformula with the other formulas in the same row, each summing up the values of the three cells above it.

However, there is a small difference with the other formulas, which is the ' +0.1 ', denoting the formula as rare, it is not like other formulas in the worksheet. Excel itself recognizes the risk of this type of formulas. This is one of the possible errors that Excel marks with a green triangle in case a formula in a row or column differs from its direct neighbors. Others are the referencing of an empty cell, and numbers formatted as text. 


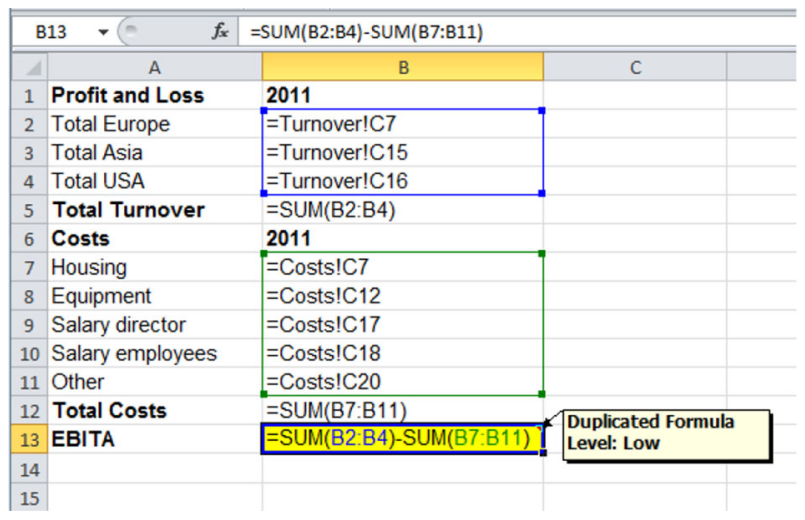

Fig. 6 A formula with duplication

In our ten spreadsheets, we encountered two cases of a Rare Formula. In both of them, a formula was marked as having a lot of duplication, turned out to differ from the other formulas in its column, while the participants stated that this was actually wrong. Thus, the smell indicated an actual error in the spreadsheet.

Note that Excel was able to mark only one of these cases as possibly dangerous: Excel spots discrepancies between directly adjacent cells, whereas one of these errors involved cells disconnected from each other.

Opinions differed on the six cases in which a common subformula were encountered. Four of the subjects understood that having the references at one place made the structure of the spreadsheets better. However the remaining two saw no harm in the duplication of formulas. This is notable, since with source code many people agree that duplication should be avoided.

With respect to the risk maps, we noticed that the current implementation of the pop up as does not yet provide enough information: It only marks the formula that shares subformulas with many formulas, but does not indicate with what cells the subformulas are shared. This resulted in participants looking through formulas in the spreadsheet to find the formulas that shared a subformula. A possible solution could be to include the list of sharing formulas in

\begin{tabular}{|c|c|c|c|c|c|}
\hline \multicolumn{2}{|r|}{$\cdot($} & \multicolumn{4}{|l|}{$f_{x}$} \\
\hline 4 & A & $B$ & C & $\mathrm{D}$ & $E$ \\
\hline 1 & Interest payable & & & & \\
\hline 2 & - Group & -20 & -33.3 & -28.9 & -23.7 \\
\hline 3 & $=--$ share of joint vent. & -5.2 & -7.2 & -6.3 & -3.7 \\
\hline 4 & Profit before taxation & $=\operatorname{SUM}(B 1: B 3)$ & $=\operatorname{SUM}(C 1: C 3)$ & $=S U M(D 1: D 3)$ & $=\operatorname{SUM}(E 1: E 3)+0.1$ \\
\hline 5 & Taxation & -22.1 & -21 & -14.7 & -13.2 \\
\hline 6 & Taxation on exc. It. & - & - & - & - \\
\hline 7 & Profit after taxation & $=\mathrm{SUM}(\mathrm{B} 4: \mathrm{B} 5)$ & ) =SUM $(C 4: C 5)$ & ) =SUM(D4:D5) & $=$ SUM(E4:E5)-0.1 \\
\hline 8 & & & & & \\
\hline 9 & & & & & \\
\hline 10 & & & & & \\
\hline
\end{tabular}

Fig. 7 The formula in E4 is a 'rare' formula 
the pop up, or create an Excel plug in that highlights the sharing formulas when a formula suffering form duplication is selected. We will address this in future work.

Conclusions Rare formulas can reveal true weaknesses and even errors in spreadsheets, and spreadsheet users agree with that.

However, the refactoring of duplicate pieces of formulas-in source code refactoring very common - is not considered to be an improvement to all spreadsheet users.

\subsubsection{Long Calculation Chain}

Findings This smell triggered most discussion with the five subjects whose spreadsheets were diagnosed with this smell.

The main reason was the fact that the risk maps do not provide enough information to understand this smell immediately. When a certain cell suffers from the Long Calculation Chain smell at the $70 \%$ level, this means that the path from this formula to the beginning of the longest calculation chain is at least 5 steps. The cells that are included in this calculation chain were not shown in the pop up. This led to spreadsheet users repeatedly stepping through formulas to check whether a formula indeed had a long calculation chain; and whether that was necessary.

Two of the subjects found that the calculation chain (in one case 10, in the other 17 steps) was indeed long, and that some restructuring would improve readability. The other three subjects found that, although the number of steps was high, this was necessary in order to calculate the needed values. We did notice that it is easier to trace and understand the different calculation steps when they are located in the same row or column. When we asked the five subjects about this, they concurred. This means there is a need for an additional metric based on the location of the cells involved in the calculation chain. We will look into this in future research.

Furthermore there is the trade off between Multiple Operations and Multiple References on one the hand, and Long Calculation Chain on the other. When discussing this phenomenon with the five subjects, we learned that they felt in need of guidance where the right balance is. Hence, better support for managing this trade off is needed. This might be done with our risk maps or with other interfaces to help users to find the proper balance between the formula smells.

Conclusions Long Calculation chains are relatively common, but are difficult to refactor for spreadsheet users. Hence more support to help users to understand and refactor this smell is necessary.

\subsubsection{Conditional Complexity}

Findings This metric was the least common in the case study, similar to the finding in the evaluation of the EUSES corpus. In the two spreadsheets in which it was located, the risk maps easily helped in locating the Conditional Complexity smell. When the users selected the cells suffering from the smell, they learned from the pop up that nested conditionals were found in the formula.

The two subjects understood and even apologized for the detected smell, stating "I know this formula is too hard, I was trying to get something to work, and then it just remained like that". Both subjects were well aware of the fact that nesting more than two conditional formulas was not such a good idea. 
Conclusions The Conditional Complexity smell is in fact already known to spreadsheet users. Apparently there is some notion among the spreadsheet users that conditional operations are complex and should be handled with some care, probably explaining the low occurrence of this smell.

\section{Refactoring Evaluation}

\subsection{Goal}

With this third evaluation, we want to obtain a preliminary understanding of the usefulness of the generated spreadsheet refactoring suggestions. To that end, the authors of the paper have re-analyzed the ten spreadsheets of Robeco and specifically investigated how smells could be refactored.

\subsection{Setup}

We use the Breviz tool to analyze the ten industrial spreadsheets of the previous evaluation and generate suggested refactorings for all smelly formulas. Subsequently, we inspected those suggestions manually for all smelly formulas. We determined whether the suggested refactoring would resolve the smell and what other impact it would have, specifically whether executing the refactoring would create new smells.

\subsection{Results}

Table 4 shows which of the six refactorings-Extract Common Subformula (ECS), Extract Subformula (ES), Group References (GR), Move References (MR), Merge Branches (MB) and Merge Formulas (MF) — could be applied to the smelly formulas in the test set, for each of the five smells. A dash (-) indicates a given refactoring can not be applied to remove that smell. Table 5 lists, for each of the smells, what refactorings need to be executed to relieve the smell.

\subsubsection{Multiple Operations}

In 81 of the 202 formulas suffering from the Multiple Operations, the Extract Common Subformula could be applied. This number is quite high; one would expect that a simple refactoring like replacing a common subformula would be something that users would perform themselves. However, we learned from the previous evaluation that improving quality of spreadsheets is not a priority. The fact that even such an obvious refactoring is not applied

Table 4 Applicable refactorings for each of the smells, on the ten spreadsheets of the second evaluation

\begin{tabular}{lccccccc}
\hline Smell & Smelly formulas & ECS & ES & GR & MR & MB & MF \\
\hline Multiple operations & 202 & 81 & 202 & - & - & - & - \\
Multiple references & 159 & - & 159 & 63 & 18 & - & - \\
Duplicated formula & 71 & - & 71 & - & - & - & - \\
Long calculation chain & 30 & - & 30 & - & - & - & - \\
Conditional complexity & 25 & 7 & 25 & - & - & 12 & - \\
\hline
\end{tabular}


Table 5 Applicable refactorings for each of the smells, on the ten spreadsheets of the second evaluation

\begin{tabular}{|c|c|c|}
\hline Smell & $\begin{array}{l}\text { Refactoring sequence needed } \\
\text { to non-smelly }\end{array}$ & \# cells \\
\hline \multirow[t]{8}{*}{ Multiple operations } & ECS & 67 \\
\hline & ECS, ES & 10 \\
\hline & ECS, ESx2 & 4 \\
\hline & ES & 47 \\
\hline & $\mathrm{ESx} 2$ & 41 \\
\hline & ESx3 & 22 \\
\hline & $\mathrm{ESx} 4$ & 11 \\
\hline & Total & 202 \\
\hline \multirow[t]{7}{*}{ Multiple references } & GR & 49 \\
\hline & $\mathrm{GR}, \mathrm{EF}$ & 14 \\
\hline & MR & 18 \\
\hline & $\mathrm{EF}$ & 35 \\
\hline & EFx2 & 23 \\
\hline & EFx3 & 20 \\
\hline & Total & 159 \\
\hline \multirow[t]{3}{*}{ Duplicated formula } & $\mathrm{EF}$ & 69 \\
\hline & Unable to refactor & 2 \\
\hline & Total & 71 \\
\hline \multirow[t]{3}{*}{ Long calculation chain } & MF & 5 \\
\hline & Unable to refactor & 25 \\
\hline & Total & 30 \\
\hline \multirow[t]{5}{*}{ Conditional complexity } & MB & 5 \\
\hline & MB,ECS & 7 \\
\hline & ES & 9 \\
\hline & $\mathrm{ESx} 2$ & 4 \\
\hline & Total & 25 \\
\hline
\end{tabular}

structurally confirms this. Of the 81 formulas, 67 were non-smelly after this refactoring, the other 14 needed additional treatment with the Extract Subformula refactoring.

\subsubsection{Multiple References}

In 63 formulas with the Multiple References smell, the references could be grouped within the formula with the Group References refactoring. In 49 of those formulas, performing this refactoring caused the formula to drop below the $70 \%$ threshold for smelliness. So this simple refactoring is quite effective. One of the possible reasons for this, is the option that Excel provides to click references while editing a formula, instead of typing their location in the formula bar. While this is a very user friendly function for people who prefer to work with the mouse, it might be the reason that individual cells are referenced instead of adjacent groups. 
In the remaining 96 smelly cells, 18 times the Move References refactoring was applicable. This means some of the referenced cells were exclusively used by the smelly formula and therefore the references could be moved without interfering with other formulas. As noted in the previous evaluation, this is actually a smell in the structure of the worksheet than in the formula itself: those references were placed a location that caused the formula to be smelly.

On the last 78 formulas we manually applied Extract Formula refactoring, causing 43 formulas to remain smelly after one step. These formulas needed two (23) or three (20) additional Extract Formula refactorings to drop below the threshold.

\subsubsection{Duplicated Formula}

The Duplicated Formula smell can only be refactored with the Extract Subformula refactoring, but the interesting fact here is that this refactoring will always impact other cells too, as there must be other cells sharing a subformula. otherwise the formula would not suffer from the Duplicated Formula smell. As described above, there are two different cases in which this smell occurs: Common Subformula and Rare Formula. In the case of a Rare Formula, the Extract Subformula does not help, since in that case the formula was never supposed to share a subformula. In the other cases, applying Extract Subformula will always relieve the smell.

\subsubsection{Long Calculation Chain}

In the second evaluation, we saw that the Long Calculation Chain smell is the hardest smell to understand, as it does not occur in one formula only, but rather concerns a group of cells. Of the 30 formulas in which this smell occurred, in only 7 of them, we could apply the Merge Formulas refactoring, as the formulas in the chain did not occur in other chains. In 5 of those 7, the refactoring could be applied without going over the threshold for Multiple References or Multiple Operations. In these cases, the trade off seemed to be not present. This is due to the fact that since these formulas only occurred in one chain, the impact of the refactoring was low. For the remaining 25 cases however, we would need additional interface support to help the user refactor formulas that occur in multiple chains. This is an interesting direction for future work.

\subsubsection{Conditional Complexity}

In 12 of the 25 formulas with the Conditional Complexity smell, branches could be merged, i.e. the Merge Branches refactoring was applicable. After the merging of those branches, in 7 cases, common subformulas could be extracted, either in the condition or in the branches. This observation also gives additional credibility to the hypothesis that such complex formulas are not created at once. If the entire conditions would be known, the spreadsheet user could have put it in one branch at creation of the formula. When multiple branches result in the same value, it seems likely some branches were added at a later time. This is corroborated by the statement of the creator of one of those formulas, who stated: "I was trying to get this formula to work". In the 13 cases on which Merge Branches could not be applied, this was the case because there were no multiple branches resulting in the same value. In that case, the smell can only be relieved by applying the Extract Subformula refactoring. In 9 of the 13 cases, the smell could be resolved with one application of Extract Subformula, in the other 4 cases, two applications were needed. 


\section{Answers to Research Questions}

With the results of the EUSES analysis and the case studies, we revisit the research questions.

$R_{1}$ What spreadsheet smells are most common, and why? In the first two evaluations we have seen that Multiple Operations and Multiple References are the most common smells, and from the second evaluation we have learned that this is often caused by the modification of a spreadsheet, sometimes under time pressure. Since there is little awareness of the risks of Multiple Operations, spreadsheet users seem not to be concerned too much about understandability and maintainability of formulas. They keep extending formulas with more operations and more references, causing formulas to become long and complicated.

$R_{2}$ To what extent do formula smells expose threats to spreadsheet quality? We found two actual faults in a spreadsheet by looking at the Duplication Smell. With respect to the other smells, the concern caught is lack of understandability. Spreadsheet users found that our current smell detection strategies reveal the formulas that are the least maintainable. These formulas will be time consuming to change, and changes made will be more error prone.

$R_{3}$ To what extent are risk maps an appropriate way to visualize spreadsheet smells? The strengths of risk maps include their simplicity and the fact that the visualization is shown within the context of the spreadsheet. Seven subjects explicitly stated they liked the risk maps, posing statements like "these colors draw my attentions to the formulas that deserve a second check". Users furthermore appreciated the different levels of the smells, allowing them to inspect the worst formulas first. For the Long Calculation smell, however, additional interface elements are needed, in order to help spreadsheet users understand the cause of the smell.

In addition to the risk maps, three of the subjects asked for a general score of the quality of their spreadsheet. Although we could provide them with the number of smells and their severity by looking into our database, an aggregation of the metrics is not provided by the current prototype. This could, for instance, be added by generating an extra worksheet in the spreadsheet in which overviews of all metrics are shown, for instance similar to the star ranking system of Cunha et al. (2012).

$R_{4}$ To what extent can formula smells be refactored by generated refactoring suggestions? In our third evaluation, we found that some smells can be refactored using the suggestions we generate. For instance the Multiple Operations smell and the Multiple References smell, especially in the case where the Group References refactoring can be applied. This refactoring does not impact other cells and is quite easy to perform. However, it might be hard for spreadsheet users themselves to perform these refactorings. In future work, we plan to investigate the possibility of automating the execution of the refactorings, especially for smells like the Long Calculation Chain that are harder to perform and balance.

\section{Discussion}

\subsection{Better Calibration of Thresholds}

In this paper, we have calibrated the thresholds based on a large set of diverse spreadsheets. However, this set stems from 2005 and the standards of spreadsheet use might have changed or could change in the future. Hence, it might be better to select a set of spreadsheets and continuously monitor their smell levels and calibrate based on that. Alternatively, we could use a smaller, more specific set, resulting in more focused guidelines. For instance, 
calibrating the metrics for a given domain or even company might improve the results, as spreadsheet formulas in different settings might have styles for formulas.

\subsection{Interacting Smells and Refactorings}

As mentioned above, some smells are related to each other, like Multiple Operations and Multiple References. Refactoring the one might also solve the other. Some other smells are inversely related like Long Calculation Chain with Multiple Operations; refactoring one might actually cause the other. In future versions of our tool, we want to take this effect into account, for instance for prioritizing the suggested refactorings.

\subsection{Named Ranges}

In the current set of smells we have not taken into account named ranges, a spreadsheet feature allowing users to assign a name to a number of cells. We encountered named ranges in one of the case studies, where a formula that summed a named range, SUM(NamedRange), was marked as having the Many Reference smells. Initially the subject did not understand why it was marked as referring to many different ranges, since there was only one reference. The named range itself however consisted of several separate ranges. This raises the question whether we think this is smelly, and why. Note that the smell is in fact related to the named range itself-it is probably not a good idea to create a named range consisting of multiple ranges - rather than to the formula referencing the named range.

\subsection{Applicability of the Risk Maps}

Our risk map visualization exhibits limitations if the smell in question addresses concerns not exclusively contained in the smelly formula itself. This explains why some subjects were dissatisfied with the pop-ups of Long Calculation Chain and Duplicated Formulas, which essentially require information from cells outside the smelly formula itself. In future research we will explore how to present smells at different levels of abstraction in one integrated view.

\subsection{Spreadsheet Evolution}

While performing the case study, subjects shared that spreadsheets tend to undergo many changes during their life time (an observation also made in Hermans et al. (2012a)) and that these changes can lead to a degradation of formula quality. This is an issue that warrants further investigation, calling for a longitudinal study of spreadsheet quality in which we could study different versions of one single spreadsheet to understand to a deeper extent how formulas become smelly.

\subsection{Threats to Validity}

A threat to the external validity of our quantitative evaluation concerns the representativeness of the EUSES Corpus spreadsheet set. This set, however, consists of 4223 spreadsheets covering 11 different domains. Furthermore, it has been used in many other studies and is collected from practice.

A threat to the external validity of our qualitative evaluation concerns the representativeness of the selected set of employees of Robeco and their spreadsheets. However other 
papers (Hendry and Green 1994; Panko 2006) report on industrial spreadsheet stories similar to the ones we found at Robeco, so their practice seems representative. Further studies are however needed to generalize our findings.

With respect to internal validity, one of the threats is the fact that we did not pick a random sample of people. This effect can be decreased by using a larger test group in future experiments. We however believe the current test group serves as a good reference group, as the persons varied in age, function and daily tasks with spreadsheets. Another threat is the fact that subjects might have cleaned up their spreadsheets before participating in the study. However, given the large number of smells we found, this does seem the case.

\section{Related Work}

Efforts related to our research include work on code smells, starting with the canonical work by Fowler (1999). His book gives an overview of code smells and corresponding refactorings. Recent efforts focused on the automatic identification of code smells by means of metrics. Marinescu (2001) for instance, uses metrics to identify suspect classes, those classes that might have design flaws. Lanza et al. (2005) explain this methodology in more detail. Alves et al. (2010) focus on a strategy to obtain thresholds for metrics from a benchmark. Olbrich et al. furthermore investigates the changes in smells over time, and discusses their impact (Olbrich et al. 2009). Moha et al. (2010) goes a step further and designed the DECOR method to automatically generate their code smell detection algorithms from specification. The CCFinder tool (Kamiya et al. 2002) specially aims at detecting clones in source code, which have similarity to our Duplicated Formula smell. The CodeCity system (Wettel and Lanza 2008; Wettel et al. 2011) presents a new technique to visualize code smells by using a 3D visualization technique, based on the metaphor of a city.

Other work related to ours include papers that provide spreadsheet design guidelines. Raffensperger (2009), for instance advises to merge references that occur only once. He furthermore states that unnecessary complex formulas with many operations and parenthesis should be avoided. Rajalingham et al. (2000) also propose guidelines to improve spreadsheet quality, which they base on principles of software engineering.

Secondly, there are papers that address common errors in spreadsheets, like (Ayalew et al. 2000; Panko 1998), together with their causes. Powell et al. for instance (Powell et al. 2009) names conditional formulas (which is one of our smells) among the top three of commonly occurring spreadsheet error categories.

Furthermore there is related work on finding anomalies on spreadsheets, for instance the work on the UCheck tool (Abraham and Erwig 2007; Chambers and Erwig 2009; Erwig 2009). UCheck determines the type of cells, and locates possible anomalies based on this type system. UCheck uses a similar visualization, with colors in the spreadsheet, to indicate found anomalies. Their follow up system UFix (Abraham and Erwig 2005) adds popup messages to explain details on the found errors.

Finally, there are papers on spreadsheet metrics, which are also aimed at locating weak points in spreadsheets. In 2004, Bregar published a paper presenting a list of spreadsheet metrics based on software metrics (Bregar 2004). He however does not provide any justification of the metrics, nor did he present an evaluation. Hodnigg and Mittermeir (2008) propose several spreadsheet metrics of which some are similar to Bregar's. Their metrics are divided into three categories: general metrics, such as the number of formulas and the number of distinct formulas; formula complexity metrics, such as the number of references per formula, and the length of the longest calculation chain; and finally metrics, such as the 
presence of scripts in, e.g., Visual Basic for Applications (VBA), user defined functions and external sources. Besides the metrics, the authors also pose the interesting suggestion to use different types of visualizations for cells with different values for the metrics. Hole et al. (2009) propose an interesting approach to analyze spreadsheets in terms of basic spreadsheet metrics, such as the number of functions used, the presence of charts and the complexity of program code constructs to predict the level of the spreadsheet creator.

We ourselves have worked on spreadsheet smells in previous work (Hermans et al. 2012a). In that paper we focused on detecting smells between worksheets, like high coupling. That paper followed our earlier work, in which we worked on the visualization of spreadsheets by means of class diagrams (Hermans et al. 2010) and dataflow diagrams (Hermans et al. 2012a).

This paper differs from our previous work by focusing on detecting smells in spreadsheet formulas. Recently, other work on spreadsheet smells has been published (Cunha et al. 2012), that aims at smells in values, such as typographical errors and values that do not follow the normal distribution. Other recent work by Badame and Dig (2012) suggests an approach to support spreadsheet users in removing formula smells by refactoring. Their refactorings are, however, not based on smells.

\section{Concluding Remarks}

The goal of this paper is to investigate the applicability of code smells to spreadsheet formulas as a means to asses and improve spreadsheet quality.

To that end we have created a list of formula smells, based on our experiences with spreadsheets, related work in spreadsheet guidelines and literature on code smells. We subsequently defined a set of metrics for detecting five formula smells and presented the visualization of these smells with the spreadsheet risk map. We have evaluated the metrics and the risk map with a qualitative and quantitative evaluation. The quantitative evaluation was performed on the spreadsheets from the EUSES corpus. The qualitative evaluation was with spreadsheets from ten professional spreadsheet users from industry.

The key contributions of this paper are as follows:

- A catalog of spreadsheet formula smells, based on code smells

- A refactoring corresponding to each smell

- A method to automatically detect these smells and suggest refactorings

- An evaluation of the formula smells on the EUSES corpus

- An evaluation of the formula smells and their refactorings on ten professional spreadsheets.

We have found that spreadsheet formula smells occur frequently, and can pose a real threat to spreadsheet understandability, and can even detect actual errors. Spreadsheet users in our qualitative evaluation found that the risk maps were a good way of indicating formula smells, and that the three thresholds helped them get a feeling of the importance of the located smells. The third evaluation showed that refactoring suggestions could resolve the detected smells with one refactoring in $59 \%$ of the cases and with no more than 4 refactorings in $95 \%$ of the cases.

The current research gives rise to several directions for future work. Firstly, the definitions of the current set of metrics could be refined; as mentioned in the evaluation section, we could split the duplication metric, and add a metric for the location of cells in a long 
calculation chain. Secondly, some smells ask for a more elaborate visualization, for instance to indicate the balance between Multiple Operations and Long Calculation Chain. Finally, more support for formula refactoring is needed.

\section{References}

Abraham R, Erwig M (2005) How to communicate unit error messages in spreadsheets. In: Proceedings of WEUSE'05, pp 1-5

Abraham R, Erwig M (2006) Inferring templates from spreadsheets. In: Proceedings of ICSE '06, pp 182-191

Abraham R, Erwig M (2007) Ucheck: a spreadsheet type checker for end users. J Vis Lang Comput 18:7195. doi:10.1016/j.jvlc.2006.06.001

Alves TL, Ypma C, Visser J (2010) Deriving metric thresholds from benchmark data. In: Proceedings of ICSM '10. IEEE Computer Society, pp 1-10

Ayalew Y, Clermont M, Mittermeir RT (2000) Detecting errors in spreadsheets. In: Proceedings of EuSpRIG '00, pp 51-62

Badame S, Dig D (2012) Refactoring meets spreadsheet formulas. In: Proceedings of ICSM '12, pp 399-409

Bregar A (2004) Complexity metrics for spreadsheet models. In: Proceedings of EuSpRIG '04, p 9

Chambers C, Erwig M (2009) Automatic detection of dimension errors in spreadsheets. J Vis Lang Comput 20:269-283

Cunha J, Fernandes JP, Mendes J, Hugo Pacheco JS (2012) Towards a catalog of spreadsheet smells. In: Proceeding of ICCSA'12. LNCS

Cunha J, Fernandes JP, Peixoto C, Saraiva J (2012) A quality model for spreadsheets. In: Proceedings of QUATIC '12, pp 231-236

Cunha J, Saraiva J, Visser J (2009) Discovery-based edit assistance for spreadsheets. In: Proceedings of VL/HCC '09, pp 233-237. IEEE

Erwig M (2009) Software engineering for spreadsheets. IEEE Softw 26:25-30. doi:10.1109/MS.2009.140

Fisher M, Rothermel G (2005) The EUSES spreadsheet corpus: a shared resource for supporting experimentation with spreadsheet dependability mechanisms. In: Proceedings of WEUSE '05, pp 47-51

Fowler M (1999) Refactoring: improving the design of existing code. Addison-Wesley Longman Publishing Co., Inc., Boston

Hendry DG, Green TRG (1994) Creating, comprehending and explaining spreadsheets: a cognitive interpretation of what discretionary users think of the spreadsheet model. Int J Human-Computer Stud 40(6):1033-1065

Hermans F, Pinzger M, van Deursen A (2010) Automatically extracting class diagrams from spreadsheets. In: Proceedings of ECOOP' 10 , pp 52-75

Hermans F, Pinzger M, van Deursen A (2011) Breviz: spreadsheet visualization and quality analysis. In: Proceedings of EuSpRIG '11, pp 63-72

Hermans F, Pinzger M, van Deursen A (2012a) Detecting and visualizing inter-worksheet smells in spreadsheets. In: Proceedings of ICSE '12, pp 441-451

Hermans F, Pinzger M, van Deursen A (2012b) Detecting code smells in spreadsheet formulas. In: Proceedings of ICSM '12, pp 409-418

Hermans F, Pinzger M, van Deursen A (2012c) Measuring spreadsheet formula understandability. In: Proceedings of EuSpRIG '12

Hodnigg K, Mittermeir R (2008) Metrics-based spreadsheet visualization: support for focused maintenance. In: Proceedings of EuSpRIG '08, p 16

Hole S, McPhee D, Lohfink A (2009) Mining spreadsheet complexity data to classify end user developers. In: Proceedings of ICDM '09. CSREA Press, pp 573-579

Kamiya T, Kusumoto S, Inoue K (2002) Ccfinder: a multilinguistic token-based code clone detection system for large scale source code. TSE 28(7):654-670

Lanza M, Marinescu R, Ducasse S (2005) Object-oriented metrics in practice. Springer-Verlag, New York, Inc., Secaucus

Marinescu R (2001) Detecting design flaws via metrics in object-oriented systems. In: Proceedings of TOOLS '01. IEEE Computer Society, pp 173-182

Moha N, Gueheneuc YG, Duchien L, Le Meur AF (2010) Decor: a method for the specification and detection of code and design smells. TSE 36(1):20-36

Nardi B, Miller J (1990) The spreadsheet interface: a basis for end user programming. In: Proceeding of the IFIP conference on human-computer interaction (INTERACT). North-Holland, pp 977-983

Olbrich S, Cruzes DS, Basili V, Zazworka N (2009) The evolution and impact of code smells: a case study of two open source systems. In: Proceedings of ESEM '09, pp 390-400 
Panko R (2006) Facing the problem of spreadsheet errors. Decis Line 37(5):8-10

Panko RR (1998) What we know about spreadsheet errors. J End User Comput 10(2):15-21

Powell S, Baker K, Lawson B (2009) Errors in operational spreadsheets: a review of the state of the art. In: Proceedings of HICCS '09. IEEE Computer Society, pp 1-8

Raffensperger J (2009) New guidelines for spreadsheets. Int J Bus Econ 2:141-154

Rajalingham K, Chadwick D, Knight B, Edwards D (2000) Quality control in spreadsheets: a software engineering-based approach to spreadsheet development. In: Proceedings HICSS '00, pp 133-143

Wettel R, Lanza M (2008) Visually localizing design problems with disharmony maps. In: Proceedings of SoftVis '08, pp 155-164

Wettel R, Lanza M, Robbes R (2011) Software systems as cities: a controlled experiment. In: Proceedings of ICSE '11, pp 551-560

Winston W (2001) Executive education opportunities. OR/MS Today 28(4)

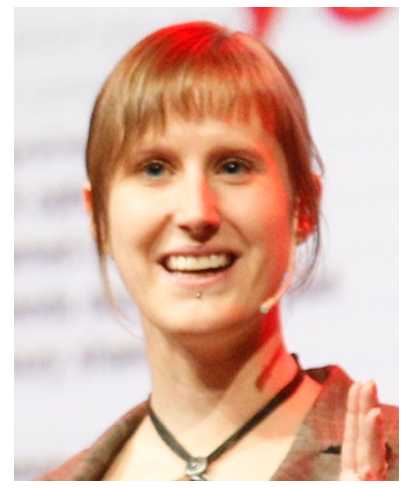

Felienne Hermans is an assistant professor at the Delft University of Technology. In January 2013, she defended her $\mathrm{PhD}$ thesis that centers around applying techniques for software to spreadsheets, in order to understand, visualize and improve them. In 2010 Felienne founded Infotron, a startup that uses the algorithms developed during the $\mathrm{PhD}$ project to analyze spreadsheet quality for large companies. In her spare time, Felienne volunteers as a referee for the First Lego League, a world wide technology competition for kids.

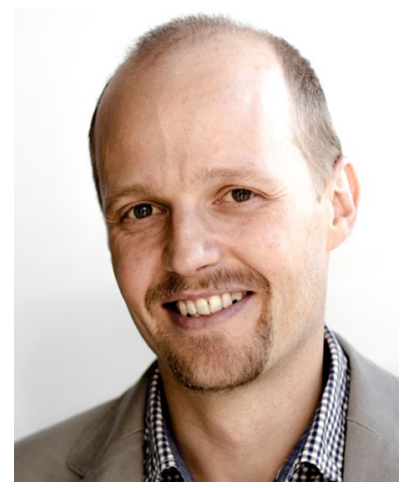

Martin Pinzger is a full professor of Software Engineering and the head of the Software Engineering Research Group at the University of Klagenfurt, Austria. His research interests are in software engineering with focus on software evolution, software design, software quality analysis, collaborative software engineering, software repository mining, software visualization, and empirical studies in software engineering. $\mathrm{He}$ received a PhD in informatics from the Vienna University of Technology in 2005. He was a Postdoc at the University of Zurich and an Assistant Professor at the Delft University of Technology from which he received tenure in 2012. In 2012, he won an NWO Vidi grant, one of the most prestigious Dutch individual research grants. In 2013, he received an ICSE 2013 ACM SIGSOFT distinguished paper award and the ICSM 2013 Most Influential Paper award. 


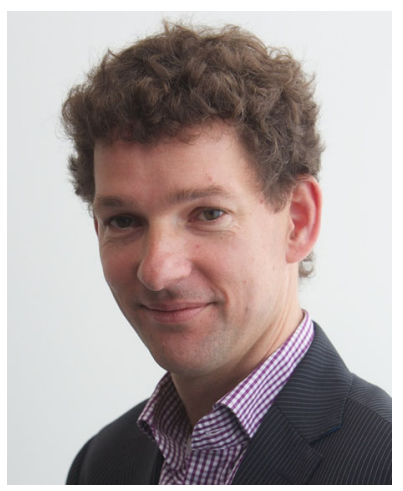

Arie van Deursen is a professor at Delft University of Technology where he is head of the Software Engineering Research Group. His research interests include software testing, software architecture, program comprehension, and end user programming. 